\title{
Capsule Commentary on Koroukian et al., Combinations of Chronic Conditions, Functional Limitations, and Geriatric Syndromes that Predict Health Outcomes
}

\author{
Amir Mohammad, MD, MPH, FACPM \\ Yale University School of Medicine and VA Connecticut Healthcare System, West Haven, CT, USA.
}

J Gen Intern Med 31(6):649

DOI: $10.1007 / \mathrm{s} 11606-016-3660-\mathrm{z}$

(c) Society of General Internal Medicine 2016

$\mathrm{K}$ oroukian et al. ${ }^{1}$ attempted to predict poor or worsening self-reported health over 2years among the elderly population based on chronic conditions, functional limitations, and geriatric syndromes, utilizing data from the US Health and Retirement Study (HRS), which includes longitudinal data characterizing multi-morbidity and health outcomes among 16,640 respondents. They focused on three outcomes: fair/ poor self-reported health status, worsening self-rated health at 2years, and 2-year mortality. The authors argued that relying solely on administrative claims data could be misleading when predicting functional limitations and geriatric syndromes among elderly.

The authors analyzed commonly experienced geriatric syndromes and functional limitations that were self or proxy reported, and limited to eight conditions as reported in HRS. They found the percent of respondents with fair/poor selfreported health status and self-rated worse health at 2 years was highest among those with lung diseases (54\%), psychiatric conditions (53\%), those with baseline limitations in Activities of Daily Living (ADLs) (80\%) or Instrumental Activities of Daily Living (IADLs) (65\%) and severe pain (77\%). However, 2-year mortality was highest among respondents with stroke (16\%), limitations in ADLs (27\%) and IADLs (16\%), and poor cognitive functioning (27\%).

Previous studies have shown that chronic diseases are common among the elderly. Barnes reported that physical activity decreases, and, conversely, inactivity increases as individual ages. $^{2}$ Over half of adults more than 65years of age report functional limitations such as walking, grasping, carrying, or pushing, and the prevalence of disability doubles in successive age groups. ${ }^{3}$ Lin et al. found that the arthritis was the greatest contributor to disability, followed by obesity, while cancer was the least important contributor to disabilities. ${ }^{4}$

Aging and chronic diseases are primary correlates of disability and compromised quality of life. The growing percentage of geriatric population is expected to be accompanied by a considerable burden on public health system, and therefore will require both intervention and innovations in decreasing the rate of chronic diseases and functional impairment. Future studies should be conducted to validate the authors' analytical approach to identifying vulnerable elders. In addition, interventional studies to assess how much of this decline is preventable and the optimal approaches to preventing decline are needed.

Corresponding Author: Amir Mohammad, MD, MPH, FACPM; Yale University School of Medicine and VA Connecticut Healthcare System, West Haven, CT, USA (e-mail: amir.mohammad@yale.edu).

\section{Compliance with Ethical Standard:}

Conflict of Interest: The author has no conflict with any of the material in this manuscript.

\section{REFERENCES}

1. Koroukian SM, Schiltz N, Warner DF, et al. Combinations of Chronic Conditions, Functional Limitations, and Geriatric Syndromes that predict health outcomes. J Gen Intern Med. 2016. doi:10.1007/s11606-016-3590-9.

2. Barnes P. Physical activity among adults: United States, 2000 and 2005. Hyattsville: US Department of Health and Human Services, CDC; 2007.

3. Mot1 RW, McAuley E. Physical activity, disability, and quality of life in older adults. Phys Med Rehabil Clin N Am. 2010;21(2):299-308. doi:10.1016/j. pmr.2009.12.006.

4. Lin SF, Beck AN, Finch BK. Trends in US older adult disability: exploring age, period, and cohort effects. Am J Public Health. 2015;102(11):2157-63. doi:10.2105/AJPH.2011.300602. 